\title{
Potentiation of delayed matching with variable delays
}

\author{
K. GEOFFREY WHITE \\ University of Otago, Dunedin, New Zealand \\ and \\ JULIE BUNNELL-MCKENZIE \\ Victoria University of Wellington, Wellington, New Zealand
}

\begin{abstract}
In a delayed matching-to-sample procedure, pigeons chose a comparison stimulus that matched a sample stimulus presented earlier in the trial. The duration of the delay between sample-stimulus presentation and comparison-stimulus presentation was either varied over five values within each session or held constant within each session but varied over five blocks of sessions. Accuracy of matching to sample was higher overall with variable delays than with delays fixed within sessions. The result indicates that remembering depends on the temporal context provided by delay intervals.
\end{abstract}

In delayed matching to sample, a sample stimulus presented on a center key is followed, after a delay interval, by a pair of comparison stimuli presented on two side keys. A response to the comparison stimulus that matches the prior sample is followed by food. Of the many variables that influence performance in delayed matching to sample, the duration of the delay interval is perhaps the most potent, in that lengthening the duration of the delay invariably leads to a reduction in matching accuracy (Blough, 1959; Roberts \& Grant, 1976; Shimp \& Moffitt, 1977; Wilkie, 1978). That is, increasing the delay-interval duration has the effect of attenuating the influence of the sample stimulus on the subsequent choice between the comparison stimuli.

Two main ways of varying delay-interval duration, intersession and intrasession variation, seem to have produced generally similar results, with matching accuracy decreasing as a negatively accelerated function of delay. Intersession manipulation of delay involves holding the delay-interval duration constant over the trials within sessions and varying duration across sessions or, more usually, across conditions. Intrasession manipulation of delay involves varying delay duration across the trials within sessions. Hence intersession manipulation involves "fixed delays," whereas intrasession manipulation involves "variable delays." Blough (1959) was perhaps the first to use variable delays. With many sessions of training, one of Blough's pigeons attained an ac-

\footnotetext{
This research was supported by grants from the University Grants Committee to K. G. White. We thank W. Edhouse and W. Attrill for their comments and assistance, and an anonymous reviewer for helpful suggestions in revising the manuscript. Reprint requests should be addressed to K. G. White, Department of Psychology, University of Otago, P.O. Box 56, Dunedin, New Zealand.
}

curacy averaging over $90 \%$ correct at a 10 -sec delay. In other studies with experienced subjects, matching accuracy is high at delays in the region of 6-20 sec (Berryman, Cumming, \& Nevin, 1963; Cumming \& Berryman, 1965; Roberts, 1980; White \& McKenzie, 1982) or longer (Grant, 1976). But with fixed delays, matching accuracy decreases to near-chance levels even at fairly short delays and remains at chance for longer delays (Harnett, McCarthy, \& Davison, 1984; Jans \& Catania, 1980; Roitblat \& Scopatz, 1983).

The results of the many studies in which matching accuracy dropped to near-chance levels with delays of a few seconds have been generally interpreted as support for the notion that the delayed matching-to-sample procedure taps short-term memory processes (Grant, 1981; Roberts \& Grant, 1976). That is, the decrement in performance to chance levels over a few seconds seems consistent with the rapid loss of information from the short-term store expected by stages-of-processing theories for both verbal (Peterson \& Peterson, 1959) and visual material (Phillips \& Baddeley, 1971).

Carter and Werner (1978) described data from a delayed matching-to-sample procedure in which delay intervals of different durations were combined in various ways within sessions. Delay intervals were generated by a probability gate queried by a time base every $1 \mathrm{sec}$. When the gate was set at .5 , for example, there was a .5 probability of a zero delay and a .5 probability of longer delays occurring on a given trial. That is, the probability of a 2sec delay was .25 , that of a 3-sec delay was .125 , and so on. When the probability of a zero-delay trial was high, matching accuracy at zero was high (about .95) and decreased to about .7 for delays of 4-5 sec. When the probability of a zero delay was low, matching accuracy was lower at zero delays (about .85) and decreased to 
about .65 at 5-sec delays. Carter and Werner's data thus indicate that interspersing long delays among short delays reduces overall accuracy, or alternatively, that a context of short delays generally facilitates performance. Unfortunately, Carter and Werner's procedure confounded relative frequency of the different delays with different delay durations, and so it is difficult to arrive at a firm conclusion from their data about the influence of variable delays on matching accuracy.

A clearer answer is provided by an experiment by Honig and Wasserman (1981), who used Nelson and Wasserman's (1978) successive matching-to-sample procedure, in which responding to a single comparison stimulus may or may not be reinforced depending upon the prior sample. Following training with delays of 0,5 , and $10 \mathrm{sec}$ within each session, Honig and Wasserman examined performance with delays of 5,10 , and $25 \mathrm{sec}$. When sessions included a 0 -sec delay, performance at 5- and 10 sec delays was more accurate than when sessions included the 25-sec delay. Honig and Wasserman's result indicates that when the duration of the delay interval varies from trial to trial within sessions, matching accuracy is determined by the context provided by the different delays.

In the present experiment, delay-interval functions for variable delays were compared to those for fixed delays. Compared to matching accuracy obtained when the duration of the delay is fixed from trial to trial within sessions, Honig and Wasserman's (1981) result suggests that accuracy in variable-delay procedures might be attenuated at short delays by the context of long delays and potentiated at long delays by the context of short delays. In other words, functions relating matching accuracy to delay may reveal an interaction between delay-interval duration and fixed-delay versus variable-delay conditions. The comparison is of theoretical interest because the interaction between delay-interval functions expected according to Honig and Wasserman's result is the opposite of that predicted by D'Amato's (1973) temporal discrimination model, as will be explained below. Furthermore, a trace decay model predicts no effect of fixed-versus variabledelay conditions. The result of the present experiment should clarify whether fixed-versus variable-delay conditions generate different functions and whether the functions interact.

\section{METHOD}

\section{Subjects}

Five adult homing pigeons with experience in various matchingto-sample procedures (White, 1983; White \& McKenzie, 1982) were maintained at $80 \%$ of their free-feeding weights. Daily sessions were conducted for each bird only if the bird's weight was within $\pm 5 \%$ of the prescribed weight. Water and grit were always available in the living cages, and supplementary feeding of mixed grain ensured maintenance of the prescribed weights.

\section{Apparatus}

A sound-attenuating experimental chamber with internal dimensions of $31 \times 34 \times 33 \mathrm{~cm}$ contained a three-key intelligence panel. The interior was painted matte black. The hopper opening was beneath the center key. An exhaust fan provided general masking noise. The three translucent response keys were $2.5 \mathrm{~cm}$ in diameter and $10 \mathrm{~cm}$ apart, center to center. Closure of microswitches mounted behind the keys required pressures of $.15 \mathrm{~N}$ for the center key and $.20 \mathrm{~N}$ for the side keys.

In-line displays were mounted behind the keys. Sample and comparison stimuli presented on the center and side keys were $.8-\mathrm{cm}$ discs of color produced by Kodak Wratten filters 74 and 73 with peak transmission at wavelengths of 538 and $576 \mathrm{~nm}$, respectively. Kodak neutral-density filters were added to the Wratten filters in order to equate brightness of the color stimuli on all three keys at $.2 \mathrm{~cd} / \mathrm{m}^{2}$. There was no houselight; the only sources of illumination were provided by the stimuli presented on the keys or by illumination of the hopper during 2-sec grain presentation. Experimental events were controlled and recorded by electromechanical and solidstate apparatus located in an adjacent room.

\section{Procedure}

Owing to their prior training in conditions similar to the present procedure, the birds did not require preliminary training and were introduced directly to the first experimental condition. Each trial in every session began with presentation of the center key illuminated by a sample-stimulus wavelength of 538 or $576 \mathrm{~nm}$. The fifth peck on the center key darkened it and initiated a delay interval. During the delay interval, the chamber was dark and responses were ineffective. The order of the sample stimuli was randomized in blocks of 10 trials within each session, with the constraint that the same sample could occur on no more than 3 consecutive trials. The delay interval terminated with onset of side-key comparison stimuli, one with the $538-\mathrm{nm}$ wavelength and the other with the 576-nm wavelength, randomly located on left and right side keys over trials. Correct side-key responses were choices of the 538-nm comparison if the prior sample was $538 \mathrm{~nm}$ or of the 576- $\mathrm{nm}$ comparison if the prior sample was $576 \mathrm{~nm}$. A single correct response darkened both keys and produced 2-sec access to grain and a dark 10 -sec intertrial interval. A single incorrect side-key response darkened both keys and initiated the dark $10-\mathrm{sec}$ intertrial interval. Each daily session comprised 80 trials, with equal numbers of presentations of the 538- and 576-nm sample stimuli for the different delay-interval durations arranged within session.

In Sessions 1-20, five different delay-interval durations were arranged in a random order over the 80 trials within each session (variable-delay condition). The delays were $.5,2,4,8$, and $20 \mathrm{sec}$. There were 16 trials at each delay in each session. In Sessions $21-95$, there were five different conditions, each lasting for 15 sessions. In each condition, just one of the aforementioned delay durations was arranged for all trials within a session (fixed-delay conditions). Each of the 5 birds was run with all five delays over the five conditions, with the order of delays for each bird randomized according to a Latin square. For Birds T1, T2, T3, T4, and T5, respectively, the fixed-delay conditions were conducted in the following orders: 20 . $2,4, .5,8 ; 8, .5,2,20,4 ; 4,20, .5,8,2 ; 2,8,20,4, .5 ; .5$, $4,8,2,20$. In Sessions $96-140$, the variable-delay procedure of Sessions 1-20 was repeated again. The order in which the various conditions was conducted thus allowed performance in the fixeddelay conditions to be compared to performance with variable delays combined over conditions conducted before and after the fixeddelay conditions.

\section{RESULTS}

For the variable-delay conditions for each bird, correct choices of comparison stimuli following each of the two sample stimuli were summed over two blocks of 15 sessions for each of the five delays. The first block included Sessions 6-20, and the second block included Sessions 126-140. Thus, proportion correct measures at 


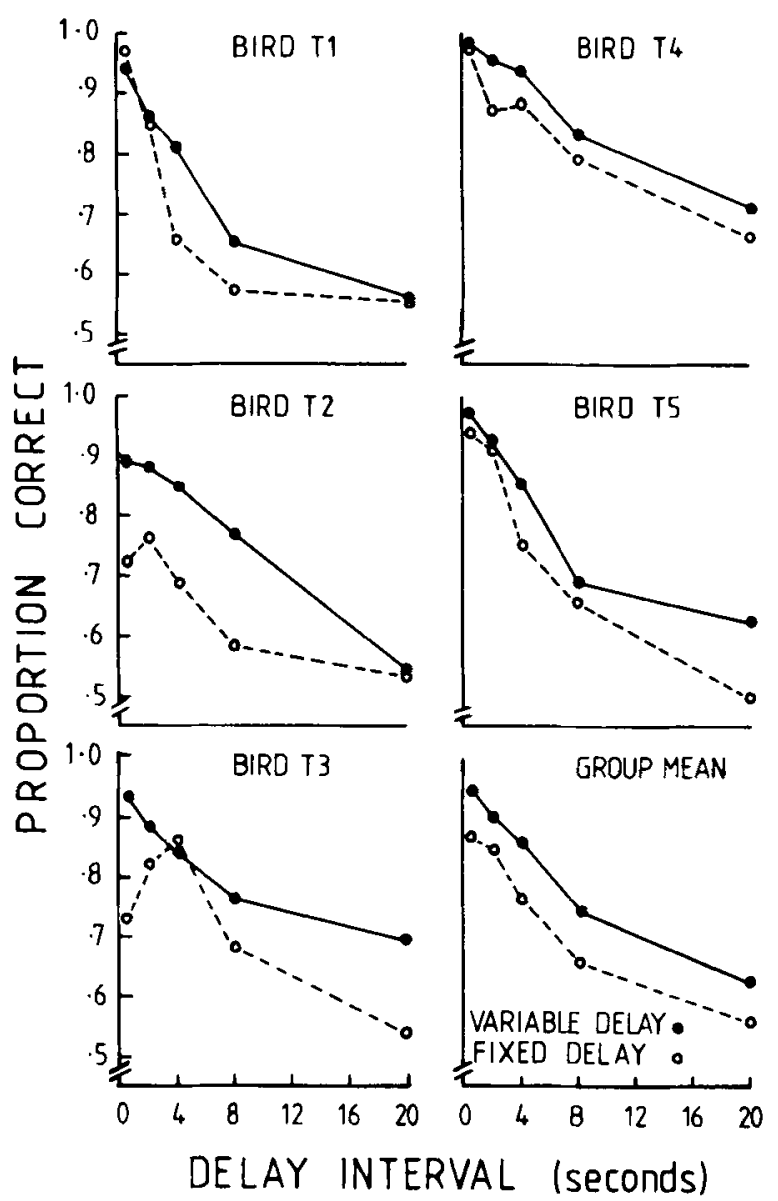

Figure 1. Proportion correct matching responses as a function of delay-interval duration for variable-delay and fixed-delay conditions.

each delay derived from these data were based on a total of 480 trials per bird. For the fixed-delay conditions, correct responses were summed for each delay over the last 6 sessions of each 15-session condition; therefore, proportion correct measures for the fixed-delay conditions were based also on a total of $\mathbf{4 8 0}$ trials per delay. Delay-interval functions for the variable-delay and fixed-delay conditions for individual birds, and also for proportions of correct responses averaged over birds, are shown in Figure 1.

Repeated-measures analysis of variance confirmed that both sets of functions for proportion correct decreased systematically with increasing delay-interval duration $[F(4,16)=39.83, p<.001]$, typical of the decrement in remembering with increasing delay obtained in previous studies of delayed matching to sample. The fixeddelay function for Bird T3 shows an irregular point at .5 -sec delay, apparently attributable to an order effect of preceding the $.5-\mathrm{sec}$ condition for this bird by the 20 -sec condition. Technically, however, the order effects are eliminated in the group-mean function by virtue of the Latin square design of orders of conditions over birds. The variable-delay function was quite stable, as indicated by the absence of a difference in proportion correct for sessions conducted before and after the fixed-delay con- ditions $[F(1,4)=3.73, p>.05]$ and by the absence of an interaction between order (before-after) and delayinterval duration $(F<1)$. Mean proportions correct in the variable-delay condition for the five delays respectively, were $.93, .89, .85, .72$, and .61 before the fixeddelay condition, and $.96, .91, .87, .76$, and .64 after the fixed-delay condition.

Matching performance in the variable-delay condition was overall more accurate than in the fixed-delay condition $[F(1,4)=19.43, p<.05]$. Accuracy was greater in the variable-delay condition at all delay intervals, and there was no interaction between condition and delayinterval duration $(F<1)$.

In order to take into account any possible fluctuations in response bias with delay interval or condition, biasfree discriminability measures at the different delays were derived from the data for each subject. The discriminability measure was that proposed by Davison and Tustin (1978) and applied by White and McKenzie (1982) to performance in delayed symbolic matching to sample. The measure is directly analogous to $\mathrm{d}^{\prime}$ of signal detection theory and is computed as half the difference between logarithms of ratios of responses to the comparison stimuli following each sample stimulus. That is,

$$
\text { Discriminability }=1 / 2[\log (\mathrm{C} 1 / \mathrm{C} 2)-\log (\mathrm{C} 3 / \mathrm{C} 4)] \text {, }
$$

where $\mathrm{Cl}$ and $\mathrm{C} 2$ are the total correct and error responses, respectively, following one sample stimulus, and $\mathrm{C} 3$ and $\mathrm{C} 4$ are the total error and correct responses, respectively, following the other sample. Discriminability measures computed according to the above equation for each subject and averaged over subjects at each delay interval are shown in Figure 2. Like the proportion correct measures, discriminability decreased systematically with increasing delay-interval duration $[F(4,16)=22.69, \mathrm{p}<.001]$ and

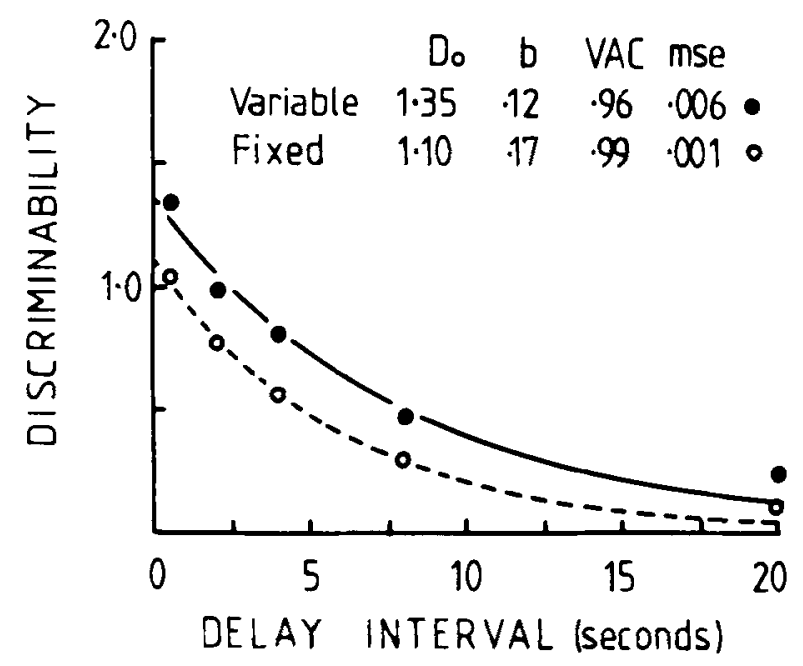

Figure 2. Mean discriminability as a function of delay-interval duration for variable-delay and fixed-delay conditions. The curves are negative-exponential functions that best fit the discriminability measures. Parameter values for the best-fitting exponential functions also are shown. 
was overall higher for the variable-delay condition than for the fixed-delay condition $[F(1,4)=18.50, p<.05]$. The data from the present study were also fitted satisfactorily by the negative exponential function used by White and McKenzie (1982), namely,

$$
\text { Discriminability }=D_{0} \cdot \exp (-b t),
$$

where $D_{0}$ is discriminability of the sample stimuli at zero delay and $b$ is the rate at which discriminability between the sample stimuli is attenuated by increasing delayinterval duration (McCarthy \& White, in press; White, 1983). The negative exponential function is a useful means of describing delayed matching-to-sample performance because it quantifies the delay-interval function in terms of the two parameters $b$ and $D_{0}$ (White, 1983). Exponential functions were fitted to the discriminability measures for each subject using a nonlinear least squares method and also to the mean discriminability measures shown in Figure 2. Estimates of the $D_{0}$ and $b$ parameters for the best-fitting functions for individual subjects and indications of how well the data were fitted by the exponential function (variance accounted for and mean squared error) are given in Table 1. The exponential function fitted the mean discriminability measures satisfactorily (Figure 2). For all birds except $T 1$, the $D_{0}$ parameter was higher for the variable-delay condition than for the fixeddelay condition. For all 5 birds, the rate at which discriminability decreased with increasing delay-interval duration (b) was greater for the fixed-delay condition. These changes in the individual functions (Table 1) are summarized by the difference between parameters for the functions best fitting mean discriminability measures in Figure 2 . The lower value for $D_{0}$ for the fixed-delay condition is consistent with the significantly lower discriminability for this condition, compared to overall discriminability for the variable-delay condition. Although each of the 5 birds showed higher values of $b$ for the fixed-delay condition than for the variable condition, this evidence of a

Table 1

Estimates of Parameter Values for Discriminability at Zero Delay $\left(D_{0}\right)$, Rate of Decrement in Discriminability (b), Variance Accounted for (VAC), and Mean Squared Error (mse) for Negative-Exponential Functions Best-Fitting Discriminability Measures for Individual Birds

\begin{tabular}{ccccc}
\hline Bird & $\mathrm{D}_{0}$ & $\mathrm{~b}$ & VAC & $\mathrm{mse}$ \\
\hline & \multicolumn{5}{c}{ Variable-Delay } \\
T1 & 1.305 & .199 & .979 & .003 \\
T2 & 1.007 & .089 & .966 & .003 \\
T3 & 1.092 & .079 & .886 & .009 \\
T4 & 1.774 & .102 & .925 & .018 \\
T5 & 1.695 & .199 & .963 & .009 \\
& & Fixed-Delay & & \\
T1 & 1.912 & .456 & .988 & .003 \\
T2 & .504 & .112 & .862 & .004 \\
T3 & .759 & .119 & .420 & .038 \\
T4 & 1.455 & .123 & .816 & .035 \\
T5 & 1.428 & .218 & .966 & .007 \\
\hline
\end{tabular}

difference in the rate of decrement is weak in view of the absence of significant interactions between delay-interval duration and condition (fixed vs. variable).

\section{DISCUSSION}

Our main finding was that delayed matching performance was overall more accurate when delay-interval duration was varied over the trials within sessions than when delays were fixed at a given duration for all trials within sessions. The overall difference in accuracy was reflected in different values of the parameter $\left(D_{0}\right)$ describing discriminability of the sample stimuli at zero delays. In particular, there was no interaction between the delay-interval functions that were virtually parallel. This result cannot easily be accommodated by theories of delayed matching, which predict that delay-interval functions should not differ when different combinations of delays are arranged (Honig \& Thompson, 1982). For example, processes hypothesized to involve trace decay (Roberts \& Grant, 1976) should not be influenced by durations of delay intervals on previous trials, except insofar as delay duration in earlier trials may modulate proactive interference by prior-trial events.

The present result also poses a problem for theories that predict an interaction between the delay-interval functions. In D'Amato's (1973) temporal discrimination model for delayed matching, the subject's task is to decide which comparison stimulus most recently appeared as the sample. When delays are fixed within sessions, the ratios of times since each sample was last seen would remain constant. Assuming that the two samples directly alternate across trials, the ratios of times for delays of $.5,2,4$, 8 , and $20 \mathrm{sec}$ are $22,7,4.5,3.25$, and 2.5 , respectively (for a 10 -sec intertrial interval). When delay intervals vary from trial to trial, temporal discrimination ratios vary from trial to trial. Given that the different delays occurred equally often on the preceding trial, average discrimination ratios for the five delays are $34.8,9.45,5.23,3.11$, and 1.85 , respectively. The temporal discrimination hypothesis thus predicts an interaction such that at .5-, 2-, and 4-sec delays, accuracy is higher with variable delays than with fixed delays, but at 8- and 20-sec delays, accuracy is lower with variable delays than with fixed delays. This interaction is clearly not in accord with the present result.

Another hypothesis predicting an interaction between the delay-interval functions is maintenance rehearsal of an instructional representation (Grant, 1981). Maintenance rehearsal may occur in varying degrees and is subject to practice effects. It is therefore plausible that rehearsal is maintained to a greater extent by variable delays than by fixed delays. But with short fixed delays, rehearsal should be at least as efficient as with short variable delays, in which case an interaction between the delay-interval functions is predicted.

Overall higher accuracy with variable delays is consistent with a variable-process account of remembering, in 
that remembering depends on the temporal context of the delays. In this respect, the present data are consistent with Honig and Wasserman's (1981) suggestion that "remembering may be an activity initiated at the time of the sample that is subject to the principles of reinforcement. This activity would be differentially rewarded by the trial outcomes" (p. 168). If we apply this suggestion to the present result, we might conclude that variable delays reinforce more persistent remembering than do fixed delays. Honig and Wasserman's data, however, are not entirely consistent with the present result. Honig and Wasserman's (Experiment 2) data are replotted in Figure 3, using a discriminability index equivalent to that applied to the present data (logarithm of the ratio of "correct" to "error" responses; McCarthy \& White, in press). Figure 3 shows that discriminability measures for 5 - and $10-\mathrm{sec}$ delays were higher when a 1 -sec delay was included in the session than when a 25 -sec delay was included. Figure 3 also shows negative exponential functions fitted to these data. (It is of interest to note that the function fitted to data for $1-, 5-$, and $10-\sec$ delays was very similar to that for the variable-delay condition in the present study). Honig and Wasserman's account for their data was that "the reinforcement schedule for remembering is presumably less favorable when the mean of the distribution of delays is long rather than short"' (pp. 168-169). The general decrement in performance with the set of long delays is reflected in the lower value of $D_{0}$. There was also a lower rate (b) at which discriminability was attenuated by increasing delay-interval duration for the long delays. Honig and Wasserman's data and their interpretation fit the present finding of lower accuracy at long delays with fixed than with variable delays. But fixed delays should have generated higher accuracy at short delays. That is, an interaction between the delay-interval functions was expected in the present data. The expected interaction is the reverse of that predicted by the temporal discrimination hypothesis.

Carter and Werner's (1978) data for different distribu-

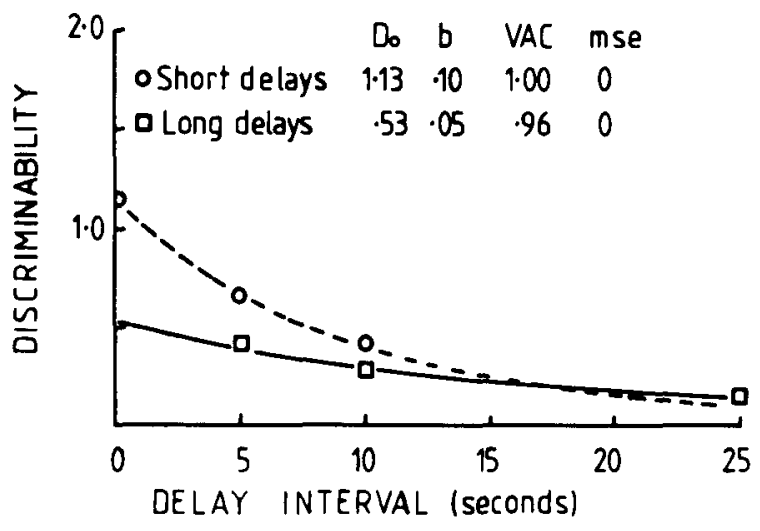

Figure 3. Discriminability measures cakculated from data described by Honig and Wasserman (1971) for sets of long delays or short delays. Curves are negative-exponential functions (parameter values for which are shown in the figure) that best fit the discriminability measures.

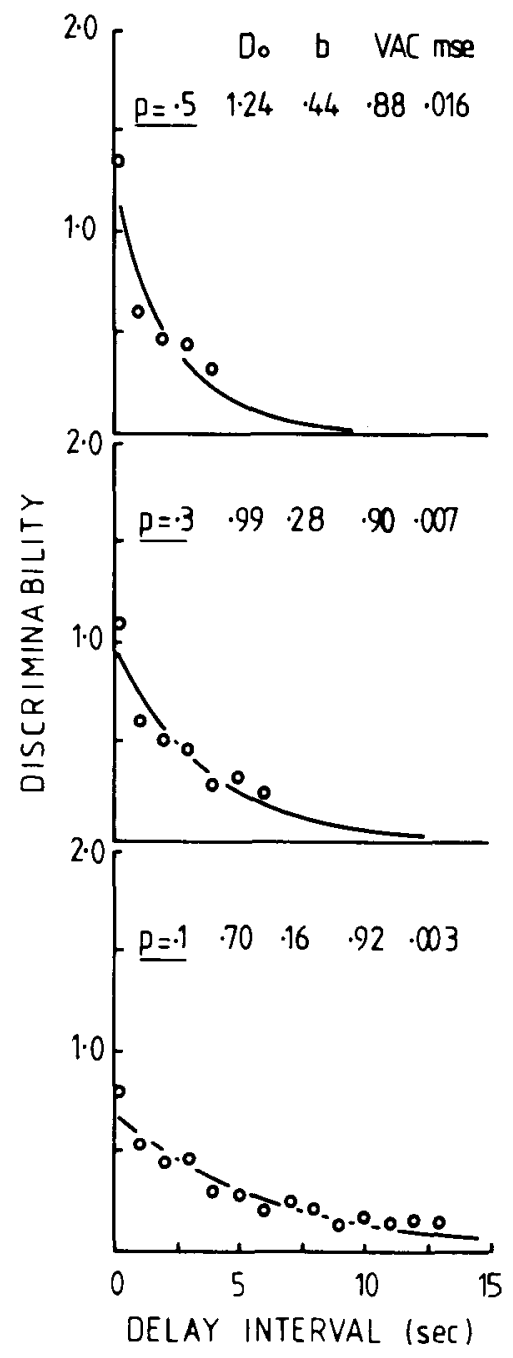

Figure 4. Discriminability measures calculated from data described by Carter and Werner (1978) for sets of conditions in which the probability of a zero delay on any trial was $.5, .3$, or .1. Curves are negative-exponential functions that best fit the discriminability measures. Parameter values for the best-fitting exponential functions also are shown.

tions of variable-delay intervals arranged within sessions are also consistent with the notion that remembering is differentially reinforced by the trial outcomes, but again are not in accord with the present result. Their data for conditions where the probability of a zero delay was .1, .3 , or .5 (Carter \& Werner, 1978, Figure 14) were converted to discriminability measures and are replotted in Figure 4. Negative exponential functions fitted to Carter and Werner's data show that, as the probability of longer delays within sessions increased, overall accuracy decreased, as indicated by a reduction in $D_{0}$. However, the changes in $D_{0}$ and $b$ in the reanalysis of Carter and Werner's data are due almost entirely to the changes in performance at zero delay. Performance at zero delay drops systematically as the probability of its occurrence decreases, resulting in the reduction in estimates for $D_{0}$ and $b$; the remaining points of the three functions are virtually indistinguishable. Performance in the high proba- 
bility (of zero-delay) conditions is considerably lower than that usually found in variable-delay conditions, instead resembling that obtained in fixed-delay conditions. This may well reflect the confounding of relative frequency and duration. In summary, Carter and Werner's (1978) data suggest that long delays in the present variable-delay condition should have had the effect of decreasing the level of accuracy at short delays with the result that the fixeddelay and variable-delay functions would interact.

In conclusion, the present data are consistent with the results reported by Carter and Werner (1978) and by Honig and Wasserman (1981) only in that they demonstrate that remembering is sensitive to the temporal context of delays. Otherwise the parallel delay-interval functions for fixed- and variable-delay conditions are not what might have been expected according to the earlier studies, and, in particular, they pose a problem for hypotheses that predict an interaction or no difference between delayinterval functions for fixed and variable delays. Perhaps the simplest account of the present result is in terms of the interpretation of the parameters of the negative exponential function fitted to the present data (White, 1983; White \& McKenzie, 1982). The fixed- and variable-delay functions did not differ in the rate of forgetting but did differ in terms of the initial discriminability of the sample stimuli at zero delay. That is, the effect of the fixeddelay condition was to attenuate sample discriminability, whereas the variable-delay condition may have resulted in greater attention being paid to the samples. That is, the variable delays resulted in reinforcing greater attention to sample stimuli. The temporal context of the delays thus had an effect on one of the two processes involved in remembering, namely sample-stimulus discriminability, without affecting the rate of forgetting.

\section{REFERENCES}

Berryman, R., Cumming, W. W., \& Nevin, J. A. (1963). Acquisition of delayed matching in the pigeon. Journal of the Experimental Analysis of Behavior, 6, 101-107.

BLough, D. S. (1959). Delayed matching in the pigeon. Journal of the Experimental Analysis of Behavior, 2, 151-160.

Carter, D. E., \& Werner, T. J. (1978). Complex learning and information processing by pigeons: A critical analysis. Joumal of the $E x$ perimental Analysis of Behavior, 29, 565-601.

Cumming, W. W., \& Berryman, R. (1965). The complex discriminated operant: Studies of matching-to-sample and related problems. In D. I. Mostofsky (Ed.), Stimulus generalization. Stanford: Stanford University Press.

D'Amato, M. R. (1973). Delayed matching and short-term memory in monkeys. In G. H. Bower (Ed.), The psychology of learning and motivation: Advances in research and theory (Vol. 7). New York: Academic Press.
Davison, M. C., \& Tustin, R. D. (1978). The relation between the generalized matching law and signal-detection theory. Journal of the Experimental Analysis of Behavior, 29, 331-336.

Grant, D. S. (1976). Effect of sample presentation time on long-delay matching in the pigeon. Learning \& Motivation, 7, 580-590.

Grant, D. S. (1981). Short-term memory in the pigeon. In N. E. Spear \& R. R. Miller (Eds.), Information processing in animals: Memory mechanisms. Hillsdale, NJ: Erlbaum.

Harnett, P., McCarthy, D., \& Davison, M. (1984). Delayed signal detection, differential reinforcement and short-term memory in the pigeon. Journal of the Experimental Analysis of Behavior, 42, 87-111.

HoniG, W. K., \& Thompson, R. K. R. (1982). Retrospective and prospective processing in animal working memory. In G. H. Bower (Ed.), The psychology of learning and motivation (Vol. 16). New York: Academic Press.

Honig, W. K., \& Wasserman, E. A. (1981). Performance of pigeons on delayed simple and conditional discriminations under equivalent training procedures. Learning \& Motivation, 12, 149-170.

Jans, J. E., \& Catania, A. C. (1980). Short-term remembering of discriminative stimuli in pigeons. Journal of the Experimental Analysis of Behavior, 34, 177-183.

MCCARTHY, D., \& WhITE, K. G. (in press). Behavioral models of delayed detection and their application to the study of memory. In M. L. Commons, J. A. Nevin, \& H. C. Rachlin (Eds.), Quantitative analysis of behavior. Vol. 5: The effect of delay and of intervening events on reinforcement value. Hillsdale, $\mathrm{NJ}$ : Erlbaum.

Nelson, K. R., \& Wasserman, E. A. (1978). Temporal factors influencing the pigeon's successive matching-to-sample performance: Sample duration, intertrial interval and retention interval. Journal of the Experimental Analysis of Behavior, 30, 153-162.

Peterson, L. R., \& Peterson, M. J. (1959). Short-term retention of individual items. Journal of Experimental Psychology, 58, 193-198.

PhilliPs, W. A., \& Baddeley, A. D. (1971). Reaction time and shortterm visual memory. Psychonomic Science, 22, 73-74.

ROBERTS, W. A. (1980). Distribution of trials and intertrial retention in delayed matching to sample with pigeons. Journal of Experimental Psychology: Animal Behavior Processes, 6, 217-237.

ROBERTS, W. A., \& GRANT, D. S. (1976). Studies of short-term memory in the pigeon using the delayed matching-to-sample procedure. In D. L. Medin, W. A. Roberts, \& R. T. Davis (Eds.), Processes of animal memory. Hillsdale, NJ: Erlbaum.

Roitblat, H. L., \& Scopatz, R. A. (1983). Sequential effects in pigeon delayed matching-to-sample performance. Journal of Experimental Psychology: Animal Behavior Processes, 9, 202-221.

Shimp, C. P., \& MoffitT, M. (1977). Short-term memory in the pigeon: Delayed-pair-comparison procedures and some results. Journal of the Experimental Analysis of Behavior, 28, 13-25.

WHITE, K. G. (1983, November). Forgetting functions in delayed matching to sample. Paper presented at the meeting of the Psychonomic Society, San Diego, CA.

White, K. G., \& MCKenZIE, J. (1982). Delayed stimulus control: Recall for single and relational stimuli. Journal of the Experimental Analysis of Behavior, 38, 305-312.

WILKIE, D. M. (1978). Delayed symbolic matching to sample in the pigeon. Psychological Record, 28, 463-469.

(Manuscript received November 5, 1984; revision accepted for publication May 20, 1985.) 